
\title{
$\angle S$ Research Suare \\ Intravenous Vitamin D Receptor Activators in Dialysis Introduction Period
}

\section{Eri Koshi-lto}

Fujita Health University https://orcid.org/0000-0003-0089-5468

Daijo Inaguma ( $\nabla$ daijo@fujita-hu.ac.jp )

https://orcid.org/0000-0002-3977-5933

\section{Shigehisa Koide}

Fujita health university

\section{Kazuo Takahashi}

Fujita health university

Hiroki Hayashi

Fujita health university

Naotake Tsuboi

Fujita health university

Midori Hasegawa

Fujita health university

\section{Yukio Yuzawa}

Fujita health university

\section{Research article}

Keywords: chronic kidney disease, CKD-MBD, haemodialysis, vitamin D, vitamin D receptor activators.

Posted Date: October 15th, 2019

DOI: https://doi.org/10.21203/rs.2.16059/v1

License: (c) (i) This work is licensed under a Creative Commons Attribution 4.0 International License.

Read Full License 


\section{Abstract}

Background Usage of vitamin D receptor activator (VDRA) for chronic kidney disease (CKD) including patients on dialysis patients has been controversial yet. It is unknown when to begin or discontinue VDRA therapy, the type of VDRA to administer, and the method of delivery, intravenous or oral for survival in CKD patients. Therefore, we examined whether intravenous or oral VDRA early after dialysis initiation affected mortality in incident dialysis patients.

Methods The study database was derived from the Aichi Cohort Study of Prognosis in Patients Newly Initiated into Dialysis (AICOPP), a multicenter, prospective, cohort analysis of 1,520 consecutive patients who began dialysis at the 17 AICOPP group centers between October 2011 and September 2013. We excluded the 262 patients who died by March 2015, 15 patients were lost to follow-up, 241 patients with unconfirmed information about use of VDRA in March 2015, and 35 patients who discontinued VDRA between dialysis initiation and March 2015. Finally, 967 patients participated in the present study. We classified the participating patients into three groups according to the usage of VDRA in March 2015: no use of VDRA (NV group), oral VDRA (OV group), and intravenous VDRA (IV group) and compared their allcause mortality.

Results There were 104 deaths during the follow-up period (NV group, 27 cases; OV group, 53 cases; IV group, 24 cases). Significant differences between the cumulative survival rates were observed for the three groups $(p=0.010)$. The IV group was associated with low all-cause mortality compared to the NV group (Hazard ratio $=0.46,95 \% \mathrm{Cl}=0.24-0.89, \mathrm{p}=0.020$ ) by multivariate Cox proportional hazard analysis using the stepwise method.

Conclusions Our study shows that early introduction of intravenous VDRA to patients on hemodialysis seems to be associated with better prognosis.

Trial Registration The trial registration no. is UMIN 000007096, registered on January 18, 2012. 298words, Text: 2967 words

\section{Background}

Chronic kidney disease-mineral and bone disorder (CKD-MBD) is one of the most important complications in patients on dialysis because it is closely associated with mortality. The treatment strategy for CKD-MBD includes dietary phosphorus restriction, phosphate binders, vitamin $\mathrm{D}$ receptor activators (VDRA), and calcimimetics. Among them, VDRA plays a central role as medication for CKDMBD. Many reports have shown vitamin D status is related to survival and native vitamin $D$ supplementation or administration of VDRA was related to better prognosis [1-5]. The beneficial results were considered to be derived from pleiotropic actions for left ventricular hypertrophy [6-9], insulin resistance [10], and the immune system [11]. On the other hand, it is possible that VDRA leads to ectopic calcification, especially vascular calcification through hypercalcemia and hyperphosphatemia [12]. Thus, use of VDRA for CKD patients has been controversial yet. 
According to Clinical practice guidelines for the management of CKD-MBD by the Japanese Society for Dialysis Therapy, dietary phosphorus restriction, the use of a calcium-containing phosphorus binder, and/or the administration of oral VDRA are recommended to control parathyroid hormone (PTH) levels in pre-dialysis. In addition, medical therapy, including serum phosphorus/calcium management and VDRA and/or cinacalcet hydrochloride are recommended to decrease the level of intact PTH[13]. Recently, Kidney Disease Improving Global Outcomes (KDIGO) 2017 Clinical Practice guidelines suggested that calcitriol and vitamin $D$ analogs should not be used routinely for adult patients with CKD G3a-G5 not on dialysis and it is reasonable to reserve the use of calcitriol and vitamin $D$ analogs for patients with CKD G4-G5 with severe and progressive hyperparathyroidism [14]. Use of VDRA for CKD patients has been controversial yet.

The serum calcitriol level decreases in the early CKD stage and is under the lower normal limit at dialysis initiation in most CKD patients [15]. In addition, vitamin D status represented by 25-hydroxyvitamin D levels is associated with pleiotropic actions in an autocrine or paracrine manner after activation to calcitriol [16]. Therefore, it would be necessary to supply native vitamin D or administer VDRA for CKD patients. However, it is unknown when to start or discontinue VDRA therapy, the type of VDRA to administer, and the method of delivery (intravenous or oral) for survival of CKD patients. Therefore, we examined the association between the timing of VDRA therapy initiation and mortality in incident dialysis patients. Moreover, we compared all-cause mortality between the three groups (no use of VDRA, oral VDRA, and intravenous VDRA).

\section{Methods}

\section{Subjects}

The study database was derived from the Aichi Cohort Study of Prognosis in Patients Newly Initiated into Dialysis (AICOPP), a multicenter, prospective, cohort analysis of 1,520 consecutive patients who began dialysis at the 17 AICOPP group centers between October 2011 and September 2013 [17]. This study was registered as UMIN000007096. Figure 1 shows patient flow. We excluded the 262 patients who died by March 2015, 15 patients lost to follow-up, 241 patients with unconfirmed information about use of VDRA in March 2015, and 35 patients who discontinued VDRA between dialysis initiation and March 2015. Finally, 967 patients participated in the present study.

\section{Patient characteristics and data at the time of dialysis initiation (baseline)}

We defined the baseline as time of dialysis initiation. The patients' body mass index (BMI) was measured at the first dialysis session, and we checked their cardiovascular history categorized as coronary artery disease, valvular heart disease, and congestive heart failure requiring hospitalization, cerebral infarction, cerebral hemorrhage, or aortic disease from medical records. and We defined comorbidity of diabetes as fasting blood glucose level $\geq 126 \mathrm{mg} / \mathrm{dL}$, random blood glucose level $\geq 200 \mathrm{mg} / \mathrm{dL}, \mathrm{HbA} 1 \mathrm{c}$ level (National Glycohemoglobin Standardization Program) $\geq 6.5 \%$, use of insulin, or use of oral hypoglycemic agents. Before the first dialysis session, blood samples were taken for laboratory testing, and the patients' 
blood pressure was measured. Aortic calcification was assessed according to the presence or absence of aortic arch calcification on plain frontal chest radiographs taken immediately before dialysis initiation. Cardiac valve calcification was assessed according to the presence or absence of a calcified aortic or mitral valve determined by B-mode echocardiography during the 1-month periods before and after dialysis initiation. Information about drug usage was also obtained from the patients' medical records. Use of VDRA and angiotensin converting enzyme inhibitor (ACEI) / angiotensin receptor blocker (ARB) were considered as medication at the time of dialysis initiation if they had been used at least 3 months before. However, other medication use refers to the drugs taken by the patients at the time of dialysis initiation.

\section{Classification according to usage of VDRA}

Prescriptions for VDRA were surveyed in March 2015 by medical records or questionnaires to other institutions. We classified the participating patients into three groups according to use of VDRA in March 2015: No use of VDRA (NV group), oral VDRA (OV group), and intravenous VDRA (IV group).

\section{Outcomes}

Survival prognosis as of September 30, 2016, was determined from the patients' medical records. For patients who were transferred to other institutions, information was obtained using a mail survey. The starting point of survival time was set in March 2015. We defined five outcomes and compared them among the three groups: 1) all-cause mortality, 2) cardiovascular (CV)-related mortality, 3) infectionrelated mortality, 4) cancer-related mortality, and 5) non-cancer-related mortality. Cardiovascular death was defined as death caused by heart failure, acute coronary syndrome, stroke, or cardiogenic sudden death.

\section{Subgroup analysis}

The main three groups were divided into five groups according to usage of VDRA at dialysis initiation and in March 2015 as: No use of VDRA at both points (Subgroup 1), use of oral VDRA in March 2015 but not at dialysis initiation (Subgroup 2), use of oral VDRA at both points (Subgroup 3), use of intravenous VDRA in March 2015 but not at dialysis initiation (Subgroup 4), and use of oral VDRA at dialysis initiation and intravenous VDRA in March 2015 (Subgroup 5). We compared all-cause mortality among the five groups.

\section{Statistical analysis}

SPSS version 24 (IBM Corporation, New York, US) and the Easy R program (Saitama Medical Center, Jichi Medical University, Saitama, Japan) [18] were used for statistical analysis. For continuous variables, patient characteristics and baseline data were compared between the three groups using analysis of variance. Fisher's exact test was used for nominal variables. Mortality rates were compared among the three groups using the log-rank test on Kaplan-Meier curves. Factors contributing to the all-cause mortality rates were examined using univariate Cox proportional hazard regression analysis. In addition 
to the three groups, factors that were significant in the univariate analysis served as explanatory variables for the multivariate Cox proportional hazard analysis using the stepwise method (i.e. comorbidity of diabetes, history of cardiovascular disease, BMI, diastolic blood pressure, aortic calcification, estimated glomerular filtration rate [eGFR], serum phosphate, serum magnesium, and use of ACEI / ARB). In addition, to test all-cause mortality among the five groups, we conducted Cox proportional hazard analysis by setting models. In stratified analyses, all-cause mortality rates were compared by univariate Cox proportional hazard models. Model 1: Adjusted for age and gender; Model 2: Adjusted for Model 1 plus comorbidity of diabetes, history of cardiovascular disease, BMI, diastolic blood pressure, aortic calcification, eGFR, serum phosphate, serum magnesium, and use of ACEI/ARB; and Model 3: Adjusted for Model 2 plus serum-adjusted calcium. Continuous variables are expressed as mean \pm standard deviation, or as median (interquartile range); categorical variables are presented as a percentage. All pvalues less than 0.05 were considered statistically significant.

\section{Results}

\section{Comparison of patient characteristics and baseline data among the three groups}

Table 1 shows patient characteristics and baseline data. The mean follow-up period was 506 days and there were no significant differences $(p=0.439)$ between the three groups (NV, OV and IV groups). The following parameters differed among the three groups: gender, comorbidity of diabetes, serum alkaline phosphatase, serum-adjusted calcium, serum-intact PTH, serum calcitriol, bicarbonate, and use of phosphate binders.

\section{Comparison of all-cause mortality}

Figure 2 shows Kaplan-Meier curves for the cumulative survival rates of the three groups. There were 104 deaths during the follow-up period (NV group, 27 cases; OV group, 53 cases; IV group, 24 cases). Significant differences between the cumulative survival rates were observed for the three groups $(p=$ 0.010).

\section{Comparison of types of mortality}

Figure 3 displays the Kaplan-Meier curves for the three groups in terms of CV-related mortality (Figure 3A), infection-related mortality (Figure 3B), cancer-related mortality (Figure 3C), and non-cancer-related mortality (Figure 3D). Significant differences between non-cancer-related mortality were observed for the three groups $(p=0.027)$. However, no significant differences between the CV-related, infection-related, and cancer-related mortality were observed for the three groups $(p=0.243, p=0.228$, and $p=0.393$, respectively).

Hazard ratios for all-cause mortality among the three groups

In the univariate regression analyses (Table 2), all-cause mortality rates were significantly lower for the IV group than for the NV group (hazard ratio $[\mathrm{HR}]=0.44,95 \%$ confidence interval $[\mathrm{Cl}]=0.25-0.76, \mathrm{p}=$ 
$0.003)$, but not significantly different for the OV group ( $\mathrm{HR}=0.75,95 \% \mathrm{Cl}=0.47-1.19, \mathrm{p}=0.225)$. In addition, all-cause mortality was associated with some factors including age, gender, history of cardiovascular disease (CVD), BMI, diastolic blood pressure, aortic calcification, eGFR, serum-adjusted calcium, serum phosphate, serum magnesium, and use of ACEls / ARBs. The results of multivariate Cox proportional hazard analysis using the stepwise method are shown in Table 3. The IV group was associated with low all-cause mortality as compared with the NV group $(\mathrm{HR}=0.46,95 \% \mathrm{Cl}=0.24-0.89, \mathrm{p}$ $=0.02$ ). In addition, high mortality was associated with advanced age, male gender, and increase in serum-adjusted calcium.

\section{Comparison of all-cause mortality and hazard ratios among the five subgroups}

Significant differences between the cumulative survival rates were observed for the five subgroups $(p=$ 0.042) (Supplementary Figure). Figure 4 shows hazard ratio (HR) for all-cause mortality among the five subgroups. All-cause mortality rates were significantly lower for subgroup 4 than subgroup 1 in model 1 $(\mathrm{HR}=0.30,95 \% \mathrm{Cl} ; 0.11-0.86, \mathrm{p}=0.02)$. Furthermore, all-cause mortality rates were significantly lower for subgroup 5 than subgroup 1 in models 1 and $2(H R=0.43,95 \% \mathrm{Cl}=0.24-0.77, p=0.004 ; H R=0.47,95 \%$ $\mathrm{Cl}=0.23-0.96, \mathrm{p}=0.038$, respectively). However, no significant differences for mortality were observed between subgroups 1 and 5 in model $3(\mathrm{HR}=0.53,95 \% \mathrm{Cl}=0.26-1.09, \mathrm{p}=0.083)$.

\section{Discussion}

The present study demonstrated that early introduction of IV VDRA to incident dialysis patients was associated with better prognosis. The features of this study were: 1 ) We could observe the effects of VDRA for mortality in the early period after dialysis initiation, 2) we could consecutively observe the clinical course from dialysis initiation to maintenance dialysis, and 3) we could conduct the study, based in real world clinical settings.

Previous studies have shown that VDRA can provide several benefits for CKD patients besides classical actions including calcium metabolism. In CKD patients, both oral and intravenous VDRA or native vitamin D were suggested to improve their prognosis $[19,20]$. The mechanisms for that are still under discussion, however the pleiotropic effects of the regulating renin-angiotensin system and reducing infectious diseases have been reported thus far [6, 21, 22]. Some papers also reported that VDRA may prevent leftventricular hypertrophy. Meanwhile, the randomized control PRIMO [23] and OPERA [24] studies, did not demonstrate that paricalcitol improves left ventricular hypertrophy in pre-dialysis patients. Therefore, this remains controversial [8,23-25]. Obi et al have reported that the early use of VDRA within at least 2 years after dialysis initiation, was associated with lower infection- related mortality in the Japanese nationwide registry data [26]. Although when VDRA should be introduced to CKD patients remains controversial, VDRA was used within at least 3.5 years after dialysis initiation in our study, similarly to the Japanese nationwide data. According to the decline of kidney function, the serum calcitriol level decreases to below the normal lower limit far earlier before dialysis initiation. Hence, our results suggested initiation of VDRA use should be considered before decline of residual kidney function. There were some problems in 
previous reports dealing with the association between VDRA or native vitamin D and survival. Most studies were conducted in an observational manner. Meanwhile, recently, Shoji et al showed that oral VDRA use for hemodialysis patients without secondary hyperparathyroidism could not improve survival rate in a large-scale randomized control trial in Japan [27]. In the study, over 900 patients were enrolled and mortality and CVD events were compared between the two groups with or without oral VDRA use. Compared to our study, the period on dialysis of the patients enrolled in the study was longer. We could not directly indicate that VDRA use improves survival because of the observational method. We also indicated that the patients, who had taken oral VDRA before initiation of dialysis and continued it, showed relatively better prognosis than who had not. Therefore, we considered that the earlier the initiation of VDRA, the greater the benefits for CKD patients.

Moreover, researchers are unsure which to choose, intravenous or oral VDRA and when to start or discontinue VDRA or native vitamin $D$ supplementation for survival in CKD patients. Native vitamin $D$ is activated by CYP2R1 and CYP27B1, in the liver and kidneys, respectively [28]. Interestingly, 25dihydroxyvitamin $\mathrm{D}$ can change to calcitriol in different types of cells including monocytes and parathyroid cells [16]. Moreover, calcitriol could act in a paracrine and autocrine manner. Some studies showed that the calcitriol produced locally could also play an important role in pleiotropic vitamin D actions $[29,30]$. Unfortunately, native vitamin D was not available in Japan because insurance did not support the use of it. Therefore, we usually use VDRA in real world clinical settings and also in the present study.

Basically, this study compared the prognosis of the three groups divided according to route of administration of VDRA and it revealed that IV VDRA could lead to better prognosis with regard to allcause mortality. However, this favorable effect of intravenous VDRA remains unclear. Considering the fact that patients chosen to intravenous group presented lower serum calcium and higher serum intact-PTH, as one of the hypotheses, the subject of our investigation was in relatively early in the course of dialysis and had not had irreversible hyperparathyroidism yet. This may be why they reacted well to high concentration of VDRA by intravenous way and their hyperthyroidism was suppressed. Although we did not collect trends of serum calcium levels except for baseline, they could have affected the prognosis because multivariate analysis for all-cause mortality was no longer significant if serum-adjusted calcium at the baseline was added to the subgroup analysis model. Previous reports showed that intravenous maxacalcitol did not comparably increase serum calcium levels [31, 32]. The reason significant effects were not observed in terms of CV-and infectious-related deaths may be due to insufficiency of power, in fact, there were merely about 10 deaths in each group.

The limitations of this study are: First, this is an observational study, and there could remain indeterminate factors regardless of using multivariate analysis. Second, because of lack of information, we could not exclude the patients who had passed away by the surveillance in March 2015. Hence, we could not clarify the relationship between VDRA use and mortality in patients with shorter survival periods after dialysis initiation. We could collect laboratory data only at baseline. Third, the criteria for initiation of dialysis and rules for use of VDRA and calcimimetics were undetermined. However, all physicians of 
participating facilities who decided to start dialysis were certified as nephrologists by the Japanese Society of Nephrology. Therefore, we assumed that there were few differences in the timing of dialysis initiation among patients.

\section{Conclusions}

In conclusion, early introduction of intravenous VDRA for patients on hemodialysis was suggested to be associated with better prognosis. Strict management of calcium and phosphate is a prerequisite. Prospective randomized control studies are needed in the future.

\section{Abbreviations}

ACEl: Angiotensin converting enzyme inhibitor

AICOPP: Aichi Cohort Study of Prognosis in Patients Newly Initiated into Dialysis

$A R B$ : angiotensin receptor blocker

BMI: Body mass index

Cl: confidence interval

$C K D$ : Chronic kidney disease

CKD-MBD: Chronic kidney disease-mineral and bone disorder

$\mathrm{CV}$ : cardiovascular

CVD: cardiovascular disease

eGFR: estimated glomerular filtration rate

$H R$ : hazard ratio

KDIGO: Kidney Disease Improving Global Outcomes

PTH: Parathyroid hormone

VDRA: Vitamin D receptor activator

\section{Declarations}

Ethics approval and consent to participate 
This study was conducted according to the Ethical Guidelines for Clinical Research by the Japanese Ministry of Health, Labor, and Welfare (created on July 30, 2003; full revision on December 28, 2004; full revision on July 31, 2008) and the Helsinki Declaration (revised in 2013), and was approved by the clinical research ethics committees at each AICOPP group facility (approval number: 20110823-3). The subjects received oral and written explanations of the purpose of the study and provided written consent. The trial registration no. is UMIN 000007096, registered on January 18, 2012.

\section{Consent for publication}

This results have not been published previously in whole or part, except in abstract form (55th ERA-EDTA Congress, Copenhagen, Denmark, 2018).

\section{Availability of data and materials}

The datasets analyzed during the current study are available from the corresponding author on reasonable request.

\section{Competing interests}

YY has received research support grants from Otsuka Pharmaceutical Co., Ltd, Kyowa Hakko Kirin Co., Ltd, and Chugai Pharmaceutical Co., Ltd. DI has received lecture fees from Ono Pharmaceutical Co., Ltd, Kyowa Hakko Kirin Co., Ltd. Other authors have nothing to declare.

\section{Funding}

The Aichi Kidney Foundation for partially funding for this study.

\section{Authors' contributions}

DI participated in the design of the study and interpretation of data. EKI, DI, and SK participated in writing the manuscript. $\mathrm{KT}, \mathrm{HH}, \mathrm{NT}, \mathrm{MH}$, and $\mathrm{YY}$ participated in analysis of data. All authors were involved in drafting, reviewing, and have approved the final manuscript for submission.

\section{Acknowledgements}

We acknowledge the support provided by the investigators and members of the AICOPP. The Aichi Kidney Foundation funded this study.

\section{References}

1.Shoben AB, Rudser KD, de Boer IH, Young B, Kestenbaum B: Association of oral calcitriol with improved survival in nondialyzed CKD. Journal of the American Society of Nephrology: JASN2008, 19(8):16131619. 
2.Kovesdy CP, Ahmadzadeh S, Anderson JE, Kalantar-Zadeh K: Association of activated vitamin $D$ treatment and mortality in chronic kidney disease. Archives of internal medicine 2008, 168(4):397-403.

3.Shoji T, Shinohara K, Kimoto E, Emoto M, Tahara H, Koyama H, Inaba M, Fukumoto S, Ishimura E, Miki T et al: Lower risk for cardiovascular mortality in oral 1alpha-hydroxy vitamin D3 users in a haemodialysis population. Nephrology, dialysis, transplantation: official publication of the European Dialysis and Transplant Association - European Renal Association 2004, 19(1):179-184.

4.Sugiura S, Inaguma D, Kitagawa A, Murata M, Kamimura Y, Sendo S, Hamaguchi K, Nagaya H, Tatematsu M, Kurata $\mathrm{K}$ et al: Administration of alfacalcidol for patients with predialysis chronic kidney disease may reduce cardiovascular disease events. Clinical and experimental nephrology 2010, 14(1):43-50.

5.Duranton F, Rodriguez-Ortiz ME, Duny Y, Rodriguez M, Daures JP, Argiles A: Vitamin D treatment and mortality in chronic kidney disease: a systematic review and meta-analysis. American journal of nephrology 2013, 37(3):239-248.

6.Li YC, Kong J, Wei M, Chen ZF, Liu SQ, Cao LP: 1,25-Dihydroxyvitamin D(3) is a negative endocrine regulator of the renin-angiotensin system. The Journal of clinical investigation 2002, 110(2):229-238.

7.Zhou C, Lu F, Cao K, Xu D, Goltzman D, Miao D: Calcium-independent and 1,25(OH)2D3-dependent regulation of the renin-angiotensin system in 1alpha-hydroxylase knockout mice. Kidney international 2008, 74(2):170-179.

8.Bodyak N, Ayus JC, Achinger S, Shivalingappa V, Ke Q, Chen YS, Rigor DL, Stillman I, Tamez H, Kroeger PE et al: Activated vitamin D attenuates left ventricular abnormalities induced by dietary sodium in Dahl salt-sensitive animals. Proceedings of the National Academy of Sciences of the United States of America 2007, 104(43):16810-16815.

9.Chen S, Law CS, Grigsby CL, Olsen K, Hong TT, Zhang Y, Yeghiazarians Y, Gardner DG: Cardiomyocytespecific deletion of the vitamin D receptor gene results in cardiac hypertrophy. Circulation 2011, 124(17):1838-1847.

10.Al-Sofiani ME, Jammah A, Racz M, Khawaja RA, Hasanato R, El-Fawal HA, Mousa SA, Mason DL: Effect of Vitamin D Supplementation on Glucose Control and Inflammatory Response in Type II Diabetes: A Double Blind, Randomized Clinical Trial. International journal of endocrinology and metabolism 2015, 13(1):e22604.

11.Sassi F, Tamone C, D’Amelio P: Vitamin D: Nutrient, Hormone, and Immunomodulator. Nutrients 2018, 10(11).

12.Wu-Wong JR, Melnick J: Vascular calcification in chronic kidney failure: role of vitamin D receptor. Current opinion in investigational drugs (London, England: 2000) 2007, 8(3):237-247. 
13.Fukagawa M, Yokoyama K, Koiwa F, Taniguchi M, Shoji T, Kazama JJ, Komaba H, Ando R, Kakuta T, Fujii $\mathrm{H}$ et al: Clinical practice guideline for the management of chronic kidney disease-mineral and bone disorder. Therapeutic apheresis and dialysis: official peer-reviewed journal of the International Society for Apheresis, the Japanese Society for Apheresis, the Japanese Society for Dialysis Therapy 2013, 17(3):247-288.

14.Group KDIGOKC-MUW: KDIGO 2017 CLINICAL PRACTICE GUIDELINE UPDATE FOR THE DIAGNOSIS, EVALUATION, PREVENTION, AND TREATMENT OF CHRONIC KIDNEY DISEASE-MINERAL AND BONE DISORDER (CKD-MBD). Kidney Int Supple 2017, 7(1):1-59.

15.John GB, Cheng CY, Kuro-o M: Role of Klotho in aging, phosphate metabolism, and CKD. American journal of kidney diseases: the official journal of the National Kidney Foundation 2011, 58(1):127-134.

16.Melamed ML, Thadhani RI: Vitamin D therapy in chronic kidney disease and end stage renal disease. Clinical journal of the American Society of Nephrology: CJASN 2012, 7(2):358-365.

17. Hishida M, Tamai H, Morinaga T, Maekawa M, Aoki T, Tomida H, Komatsu S, Kamiya T, Maruyama S, Matsuo S et al: Aichi cohort study of the prognosis in patients newly initiated into dialysis (AICOPP): baseline characteristics and trends observed in diabetic nephropathy. Clinical and experimental nephrology 2016, 20(5):795-807.

18.Kanda Y: Investigation of the freely available easy-to-use software 'EZR' for medical statistics. Bone marrow transplantation 2013, 48(3):452-458.

19.Naves-Diaz M, Alvarez-Hernandez D, Passlick-Deetjen J, Guinsburg A, Marelli C, Rodriguez-Puyol D, Cannata-Andia JB: Oral active vitamin D is associated with improved survival in hemodialysis patients. Kidney international 2008, 74(8):1070-1078.

20.Teng M, Wolf M, Ofsthun MN, Lazarus JM, Hernan MA, Camargo CA, Jr., Thadhani R: Activated injectable vitamin D and hemodialysis survival: a historical cohort study. Journal of the American Society of Nephrology: JASN2005, 16(4):1115-1125.

21.Grubler MR, Gaksch M, Kienreich K, Verheyen N, Schmid J, BW OH, Richtig G, Scharnagl H, Meinitzer A, Pieske B et al: Effects of Vitamin D Supplementation on Plasma Aldosterone and Renin-A Randomized Placebo-Controlled Trial. Journal of clinical hypertension (Greenwich, Conn) 2016, 18(7):608-613.

22.Li YC: Discovery of vitamin D hormone as a negative regulator of the renin-angiotensin system. Clinical chemistry 2014, 60(3):561-562.

23.Thadhani R, Appelbaum E, Pritchett Y, Chang Y, Wenger J, Tamez H, Bhan I, Agarwal R, Zoccali C, Wanner $\mathrm{C}$ et al: Vitamin D therapy and cardiac structure and function in patients with chronic kidney disease: the PRIMO randomized controlled trial. Jama 2012, 307(7):674-684. 
24.Wang AY, Fang F, Chan J, Wen YY, Qing S, Chan IH, Lo G, Lai KN, Lo WK, Lam CW et al: Effect of paricalcitol on left ventricular mass and function in CKD-the OPERA trial. Journal of the American Society of Nephrology: JASN2014, 25(1):175-186.

25.Tamez H, Zoccali C, Packham D, Wenger J, Bhan I, Appelbaum E, Pritchett Y, Chang Y, Agarwal R, Wanner $\mathrm{C}$ et al: Vitamin $D$ reduces left atrial volume in patients with left ventricular hypertrophy and chronic kidney disease. American heart journal 2012, 164(6):902-909.e902.

26.Obi Y, Hamano T, Wada A, Tsubakihara Y: Vitamin D Receptor Activator Use and Cause-specific Death among dialysis Patients: a Nationwide Cohort Study using Coarsened Exact Matching. Scientific reports 2017, 7:41170.

27.Investigators TJ-D: Effect of Oral Alfacalcidol on Clinical Outcomes in Patients Without Secondary Hyperparathyroidism Receiving Maintenance Hemodialysis The J-DAVID Randomized Clinical Trial. Jama 2018, 320(December 11):2325 - 2334.

28.Thacher TD, Clarke BL: Vitamin D insufficiency. Mayo Clinic proceedings 2011, 86(1):50-60.

29.Dusso AS: Kidney disease and vitamin D levels: 25-hydroxyvitamin D, 1,25-dihydroxyvitamin D, and VDR activation. Kidney international supplements 2011, 1(4):136-141.

30.Gunta SS, Thadhani RI, Mak RH: The effect of vitamin D status on risk factors for cardiovascular disease. Nature reviews Nephrology 2013, 9(6):337-347.

31. Honda H, Koiwa F, Ogata H, Shishido K, Sekiguchi T, Michihata T, Ogawa H, Mukai M, Takahashi K, Suzuki R et al: Active vitamin D analogs, maxacalcitol and alfacalcidol, as maintenance therapy for mild secondary hyperparathyroidism in hemodialysis patients - a randomized study. International journal of clinical pharmacology and therapeutics 2014, 52(5):360-368.

32.Akizawa T, Akiba T, Hirakata H, Kinugasa E, Tominaga Y, Fukagawa M, Yokoyama K, Zhang W, Linde PG, Suzuki M: Comparison of paricalcitol with maxacalcitol injection in Japanese hemodialysis patients with secondary hyperparathyroidism. Therapeutic apheresis and dialysis: official peer-reviewed journal of the International Society for Apheresis, the Japanese Society for Apheresis, the Japanese Society for Dialysis Therapy 2015, 19(3):225-234.

\section{Tables}

Table 1. Baseline characteristics and laboratory data 


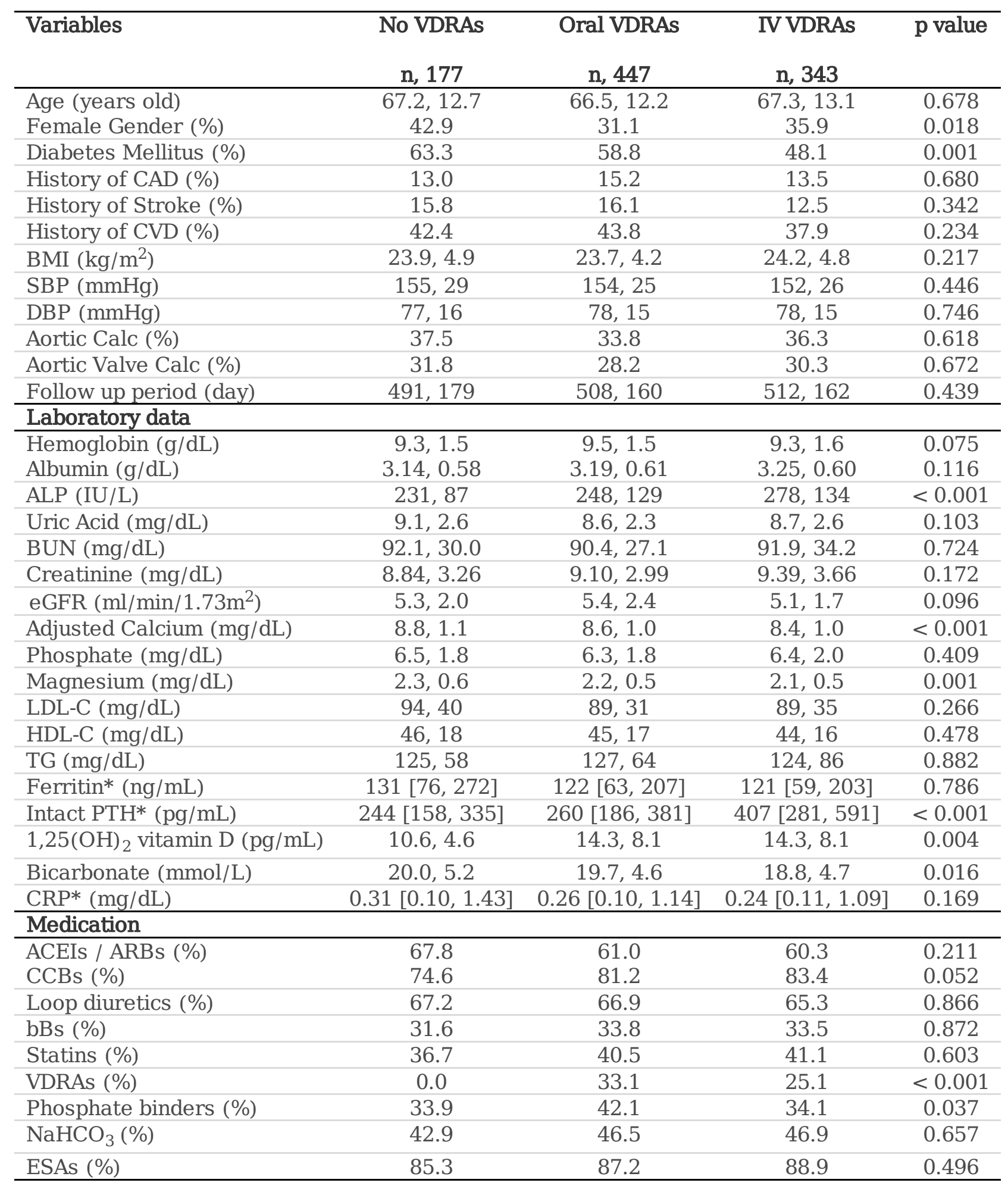

Mean, standard deviation, $*$ Median [1 ${ }^{\text {st }}$ quartile, $3^{\text {rd }}$ quartile]

VDRA; vitamin D receptor activator, IV; intravenous, CAD; coronary artery disease, CVD; cardiovascular disease, BMI; body mass index, SBP; systolic blood pressure, DBP; diastolic blood pressure, Calc; calcification, ALP; alkaline phosphatase, BUN; blood urea nitrogen, eGFR; estimated glomerular filtration rate, LDL-C; low density lipoprotein cholesterol, HDL-C; 
high density lipoprotein cholesterol, TG; triglyceride, PTH; parathyroid hormone, CRP; C reactive protein, ACEI; angiotensin converting enzyme, ARB; angiotensin receptor blocker, CCB, calcium channel blocker, bB; b blocker, VDRA; vitamin D receptor activator, ESA; erythropoiesis stimulating agent

Table 2. Associations of variables with all-cause mortality according to the univariate Cox proportional hazard regression analysis 


\begin{tabular}{lccc}
\hline Variables & Hazard Ratio & $95 \%$ CI & p value \\
\hline IV VDRAs vs No VDRAs & 0.44 & $0.25-0.76$ & 0.003 \\
Oral VDRAs vs No VDRAs & 0.75 & $0.47-1.19$ & 0.225 \\
\hline Age $(10$ years old $)$ & 2.06 & $1.68-2.53$ & $<0.001$ \\
\hline Female Gender & 0.54 & $0.34-0.85$ & 0.005 \\
\hline Diabetes Mellitus & 1.12 & $0.76-1.66$ & 0.560 \\
\hline History of CAD & 1.46 & $0.90-2.38$ & 0.129 \\
\hline History of Stroke & 1.59 & $0.99-2.54$ & 0.054 \\
\hline History of CVD & 2.07 & $1.40-3.05$ & $<0.001$ \\
\hline BMI $\left(1 \mathrm{~kg} / \mathrm{m}^{2}\right)$ & 0.93 & $0.88-0.98$ & 0.005 \\
\hline SBP $(10 \mathrm{mmHg})$ & 0.99 & $0.93-1.06$ & 0.801 \\
\hline DBP $(10 \mathrm{mmHg})$ & 0.90 & $0.91-1.00$ & 0.050 \\
\hline Aortic Calc & 1.87 & $1.28-2.75$ & 0.001 \\
\hline Aortic Valve Calc & 1.39 & $0.90-2.16$ & 0.143 \\
\hline Hemoglobin $(1 \mathrm{~g} / \mathrm{dL})$ & 1.10 & $0.97-1.26$ & 0.131 \\
\hline Albumin $(1 \mathrm{~g} / \mathrm{dL})$ & 0.82 & $0.60-1.12$ & 0.215 \\
\hline ALP $(10 \mathrm{IU} / \mathrm{L})$ & 1.01 & $0.99-1.02$ & 0.289 \\
\hline Uric Acid $(\mathrm{mg} / \mathrm{dL})$ & 1.00 & $0.93-1.09$ & 0.945 \\
\hline BUN $(10 \mathrm{mg} / \mathrm{dL})$ & 1.02 & $0.96-1.09$ & 0.476 \\
\hline Creatinine $(1 \mathrm{mg} / \mathrm{dL})$ & 0.86 & $0.80-0.93$ & $<0.001$ \\
\hline eGFR $\left(1 \mathrm{ml} / \mathrm{min} / 1.73 \mathrm{~m}^{2}\right)$ & 1.09 & $1.02-1.16$ & 0.010 \\
\hline Adjusted Calcium $(1 \mathrm{mg} / \mathrm{dL})$ & 1.38 & $1.14-1.67$ & 0.001 \\
\hline Phosphate $(1 \mathrm{mg} / \mathrm{dL})$ & 0.83 & $0.74-0.94$ & 0.003 \\
\hline Magnesium $(1 \mathrm{mg} / \mathrm{dL})$ & 1.53 & $1.07-2.19$ & 0.020 \\
\hline LDL-C $(10 \mathrm{mg} / \mathrm{dL})$ & 0.96 & $0.90-1.03$ & 0.220 \\
\hline HDL-C $(10 \mathrm{mg} / \mathrm{dL})$ & 1.11 & $0.98-1.24$ & 0.101 \\
\hline TG $(10 \mathrm{mg} / \mathrm{dL})$ & 1.01 & $0.98-1.03$ & 0.538 \\
\hline Ferritin* $(1 \mathrm{ng} / \mathrm{mL})$ & 1.00 & $0.99-1.00$ & 0.917 \\
\hline Intact PTH* $(1 \mathrm{pg} / \mathrm{mL})$ & 1.00 & $0.99-1.00$ & 0.180 \\
\hline Bicarbonate $(1 \mathrm{mmol} / \mathrm{L})$ & 1.03 & $0.99-1.08$ & 0.193 \\
\hline CRP* $(1 \mathrm{mg} / \mathrm{dL})$ & 1.03 & $0.98-1.07$ & 0.229 \\
\hline ACEIs / ARBs & 0.64 & $0.44-0.94$ & 0.023 \\
\hline CCBs & 1.09 & $0.65-1.80$ & 0.754 \\
\hline Loop diuretics & 1.15 & $0.76-1.75$ & 0.503 \\
\hline bBs & 1.00 & $0.66-1.50$ & 0.989 \\
\hline Statins & 0.72 & $0.47-1.08$ & 0.108 \\
\hline Phosphate binders & 0.70 & $0.46-1.07$ & 0.096 \\
\hline NaHCO 3 & 0.97 & $0.66-1.43$ & 0.882 \\
\hline ESAs & 0.96 & $0.54-1.72$ & 0.901 \\
\hline & & & \\
\hline
\end{tabular}

CI; confidence interval, IV; intravenous, VDRA; vitamin D receptor activator, CAD; coronary artery disease, CVD; cardiovascular disease, BMI; body mass index, SBP; systolic blood pressure, DBP; diastolic blood pressure, Calc; calcification, ALP; alkaline phosphatase, BUN; blood urea nitrogen, eGFR; estimated glomerular filtration rate, LDL-C; low density lipoprotein cholesterol, HDL-C; high density lipoprotein cholesterol, TG; triglyceride, PTH; parathyroid hormone, CRP; C reactive protein, ACEI; angiotensin converting enzyme, ARB; angiotensin receptor blocker, CCB, calcium channel blocker, bB; b blocker, ESA; erythropoiesis stimulating agent 
Table 3. Associations of variables with all-cause mortality according to the multivariate Cox proportional hazard regression analysis

\begin{tabular}{lccc}
\hline Variables & Hazard Ratio & $95 \%$ CI & p value \\
\hline IV VDRAs vs No VDRAs & 0.46 & $0.24-0.89$ & 0.022 \\
Age $(10$ years old) & 2.04 & $1.56-2.67$ & $<0.001$ \\
\hline Female Gender & 0.42 & $0.23-0.77$ & 0.005 \\
\hline Adjusted Calcium $(1 \mathrm{mg} / \mathrm{dL})$ & 1.49 & $1.13-1.96$ & 0.005 \\
\hline
\end{tabular}

CI; confidence interval, IV; intravenous, VDRA; vitamin D receptor activator

Adjusted for DM, history of CVD, BMI, DBP, aortic calcification, eGFR, serum phosphate, serum magnesium, and use of ACEIs / ARBs

DM; diabetes mellitus, CVD; cardiovascular disease, BMI; body mass index, DBP; diastolic blood pressure, eGFR; estimated glomerular filtration rate, ACEI, angiotensin converting enzyme inhibitor, ARB; angiotensin receptor blocker

\section{Figures}


Figure 1

$$
\text { Incident dialysis patients at the AICOPP facilities }(\mathrm{n}=1,520)
$$

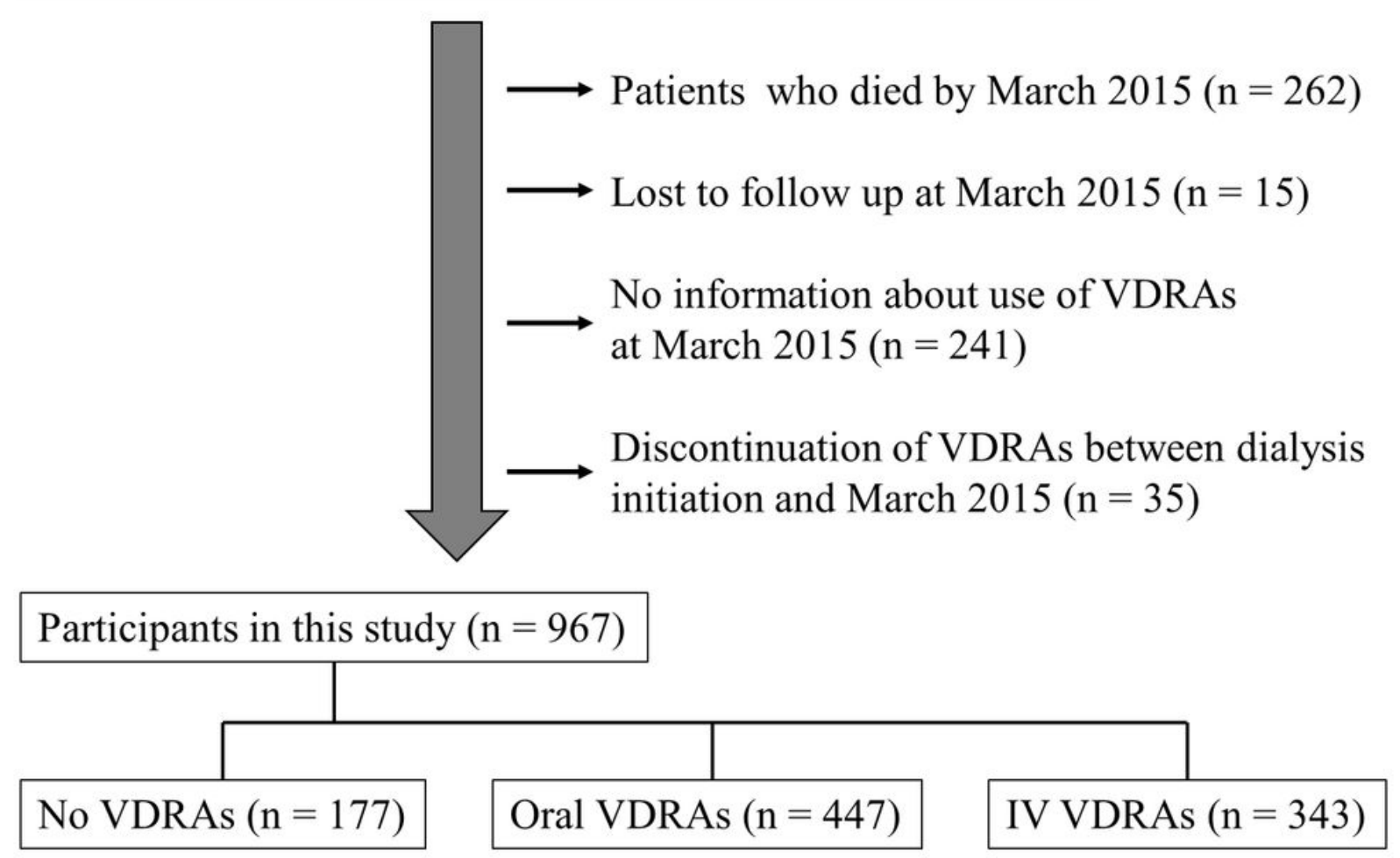

Figure 1

Participated patients flow. 
Figure 2

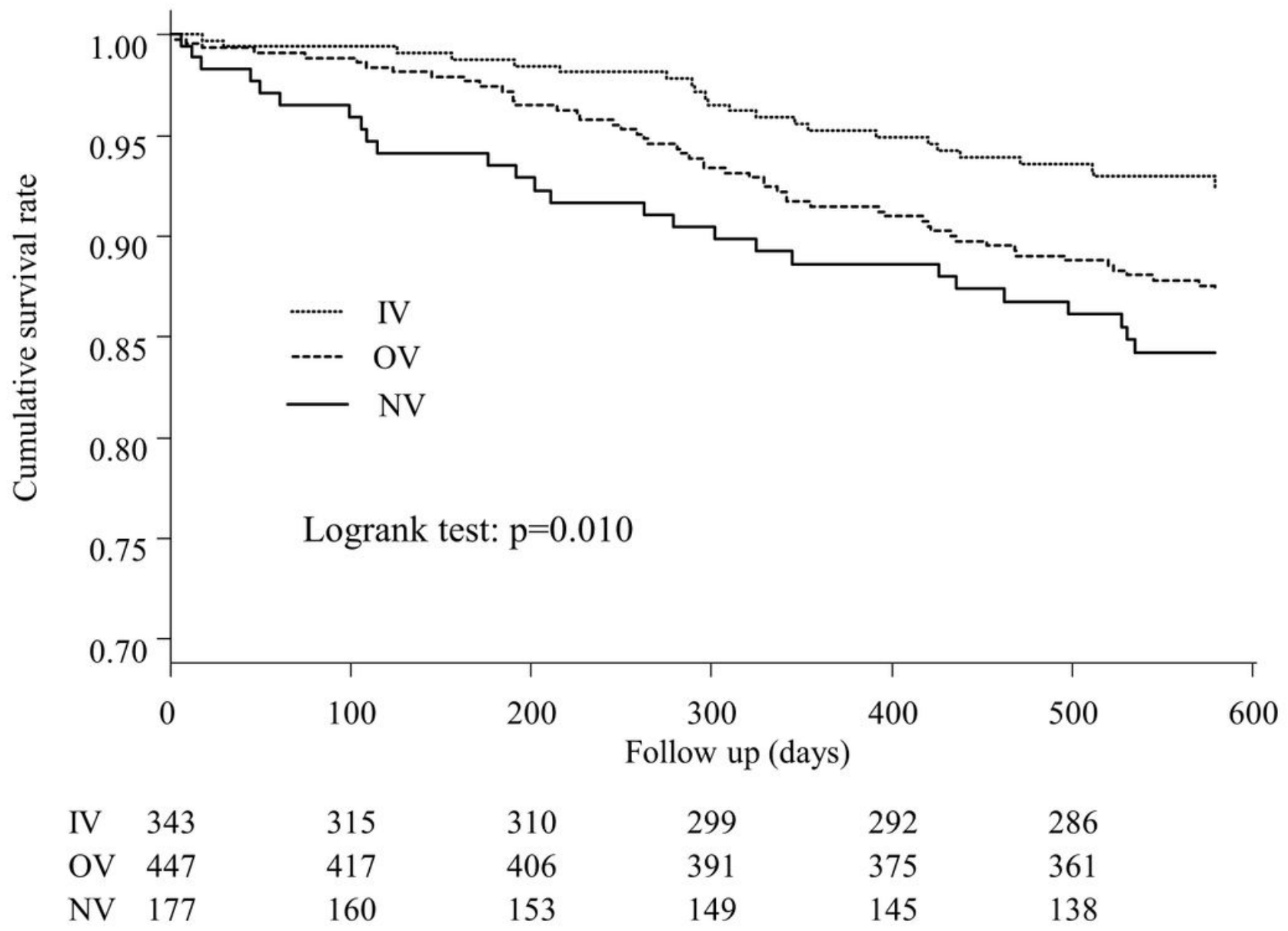

Figure 2

Kaplan-Meier curves for the cumulative survival between the three groups. Significant differences between the cumulative survival rates were observed for the three groups $(P=0.010)$. IV; intravenous VDRA group, OV; oral VDRA group, NV; no VDRA group 
Figure 3

A

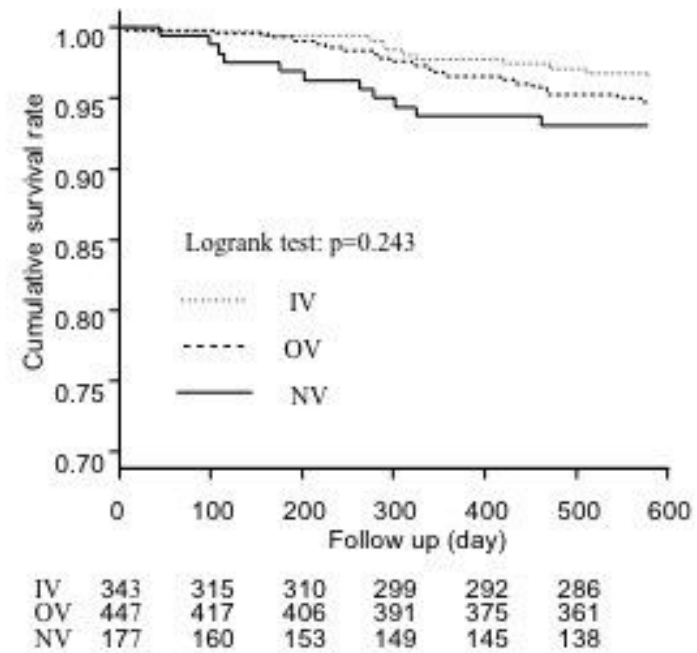

C

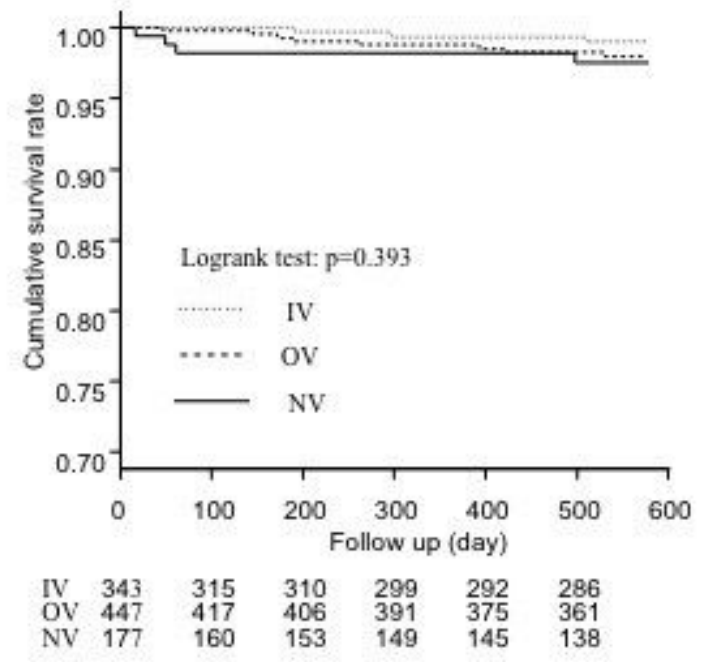

B

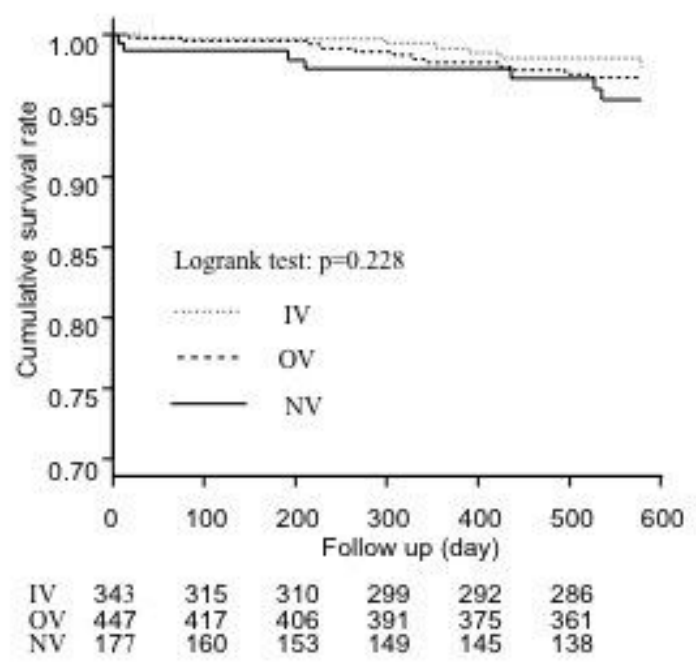

$\mathrm{D}$

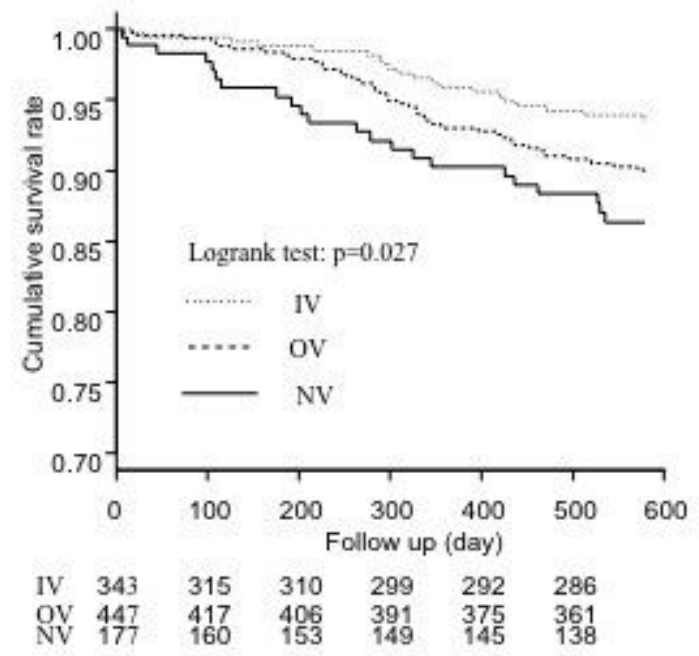

\section{Figure 3}

Kaplan-Meier curves for the cumulative survival for each cause of death between the three groups. $A$ : Comparison of cardiovascular related mortality, B: Comparison of infection related mortality, C: Comparison of cancer related mortality, D: Comparison of non-cancer related mortality Significant differences between the non-cancer-related mortality were observed for the three groups $(P=0.027)$. IV; intravenous VDRA group, OV; oral VDRA group, NV; no VDRA group 
Figure 4

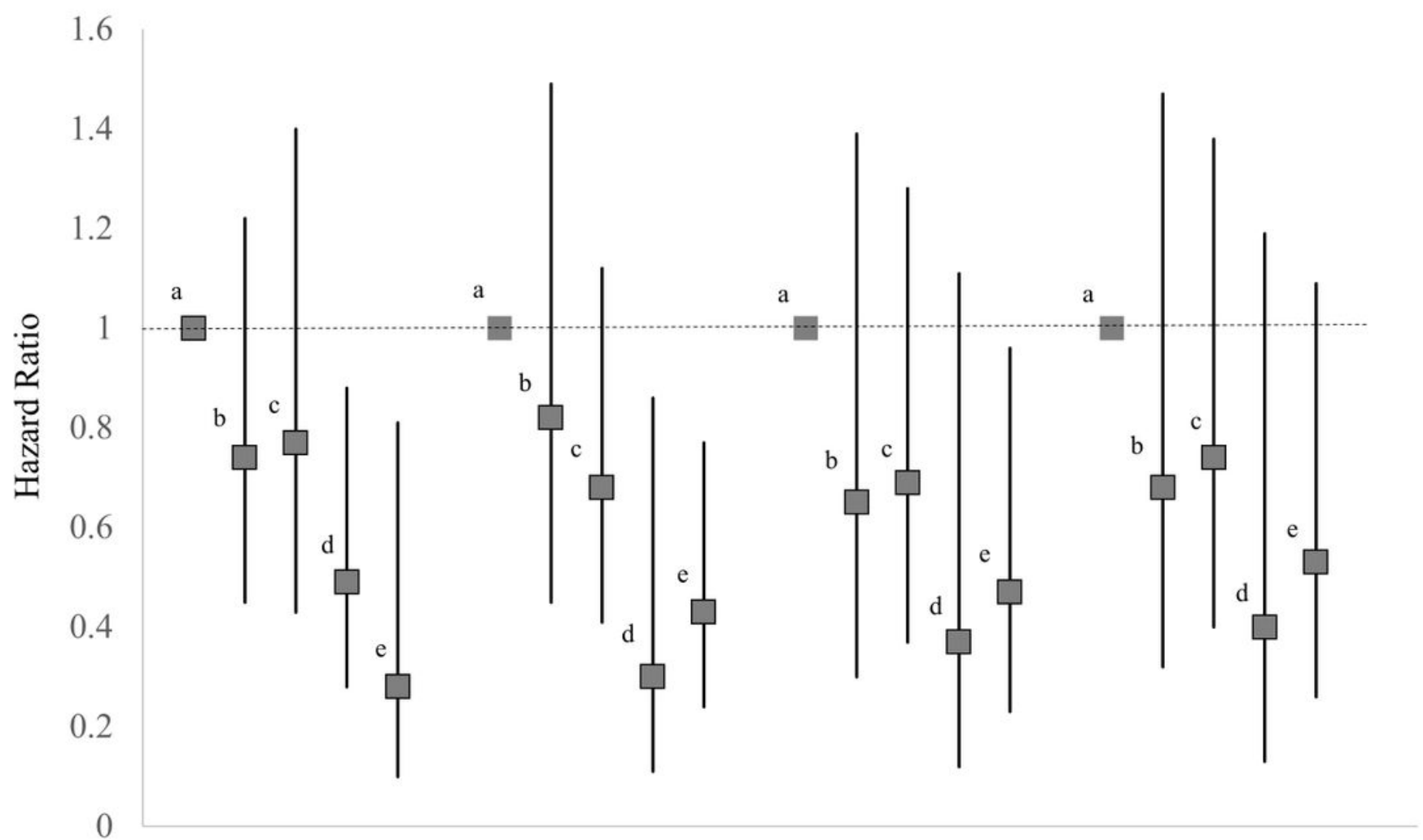

Unadjusted

Model 1

Model 2

Model 3

\section{Figure 4}

Hazard ratio for all-cause mortality among the five subgroups. All-cause mortality rates were significantly lower for the Subgroup 4 than the Subgroup 1 in model $1(\mathrm{HR}=0.30,95 \% \mathrm{Cl}=0.11-0.86, \mathrm{P}=0.024)$. Allcause mortality rates were significantly lower for the Subgroup 5 than the Subgroup 1 in model 1 and 2 $(\mathrm{HR}=0.43,95 \% \mathrm{Cl}=0.24-0.77, \mathrm{P}=0.004, \mathrm{HR}=0.47,95 \% \mathrm{Cl}=0.23-0.96, \mathrm{P}=0.038$, respectively $)$. a: no use of VDRA at the both of two points (Subgroup 1) b: use of oral VDRA in March 2015 not at dialysis initiation (Subgroup 2) c: use of oral VDRA at the both of two points (Subgroup 3), d: use of intravenous VDRA in March 2015 not at dialysis initiation (Subgroup 4) e: use of oral VDRA at dialysis initiation and intravenous VDRA in March 2015 (Subgroup 5) Model 1: adjusted for age and gender Model 2: adjusted for Model 1 plus comorbidity of diabetes, history of cardiovascular disease, BMI, diastolic blood pressure, aortic calcification, eGFR, serum phosphate, serum magnesium, and use of ACEI / ARB Model 3: adjusted for Model 2 plus serum adjusted calcium.

\section{Supplementary Files}


This is a list of supplementary files associated with this preprint. Click to download.

- SupplementaryFigure.TIF 